\title{
Comment
}

Cerebrovascular Diseases

Cerebrovasc Dis 2009;27:411-412

DOI: $10.1159 / 000209242$

\section{Intraventricular Hemorrhage: Harmful Effect Is Not from the Pressure Alone}

Kiwon Lee, Jan Claassen

Neurology and Neurosurgery, Columbia University, College of Physicians and Surgeons, New York, N.Y., USA

For patients suffering from acute intracranial hemorrhage (ICH), there is a growing body of evidence suggesting that extension of the hemorrhage into the ventricular system is associated with poor outcome [1, 2]. A study by Hemphill et al. [3] demonstrated that Glasgow Coma Scale (GCS) score, age greater than or equal to 80 years, ICH hematoma volume (measured by the $\mathrm{ABC} / 2$ method), infratentorial hemorrhage, and intraventricular hemorrhage (IVH) were independent predictors of poor outcome for patients with ICH measured by 30 -day mortality rate. Recent data presented by Hallevi et al. [4] showed that nearly half of all spontaneous ICH cases were accompanied by IVH, and that those with IVH were twice as likely to have a poor outcome. What are the proposed mechanisms leading to such poor outcomes in patients with IVH? Acute IVH is often thought to lead to acute intracranial hypertension and obstructive hydrocephalus, which then exerts pressure onto the reticular activating system resulting in a depressed level of arousal. Elevated intracranial pressure (ICP) due to IVH and hydrocephalus may also contribute to lowered cerebral perfusion pressure, further increasing the damage of the brain parenchyma. Is the presence of acute hemorrhage in the ventricular system the sole factor resulting in these deleterious effects? Neuropsychological data by Hütter et al. [5] have shown that patients with IVH suffer from significant cognitive deficits even after the blood in the ventricles has completely disappeared. This suggests that the harmful effects of intraventricular blood may not only be due to the mere presence of blood in the ventricles.

In this issue of Cerebrovascular Diseases, Ziai et al. [6] report a secondary, post-hoc analysis of data obtained during a randomized controlled trial of urokinase (UK) versus placebo for the treatment of IVH. The authors present longitudinal exploratory data of 11 patients with external ventricular drains (EVD) who were randomized to either the UK or the placebo arm of the trial. The authors reviewed a total of 472 ICP measurements in 11 patients during the entire period of having EVD placement. The primary objective of the analysis was to explore the frequency and significance of ICP elevation for patients suffering from IVH. The study subjects were patients with acute (less than $48 \mathrm{~h}$ of onset) and severe IVH requiring an EVD for the treatment of obstructive hydrocephalus. The authors demonstrated that significant intracranial hypertension, defined as ICP $>20 \mathrm{~mm} \mathrm{Hg}$, only occurred in $14 \%$ of all 472 measurements. When patients presented with IVH and obstructive hydrocephalus requiring an EVD, the initial ICP was not commonly elevated in this particular cohort of severe IVH patients.

It is noteworthy to remember that in this study patients with intraparenchymal hematoma volume greater than $30 \mathrm{ml}$, aneurysmal or arteriovenous malformation-related $\mathrm{ICH}$, and the presence of significant coagulopathy were excluded. ICP crisis is often observed in patients that were excluded in this study. For instance, a small caudate hemorrhage less than $30 \mathrm{ml}$ in hematoma volume with IVH may or may not have refractory intracranial hypertension. High Hunt and Hess grade and Fisher group 3 acute subarachnoid hemorrhage patients with IVH are notorious for high ICP which is often refractory to conventional therapies. Therefore, it should be stressed that the findings of this study should not translate into a notion that all patients with IVH and obstructive hydrocephalus do not have elevations in ICP. As the authors pointed out, having the ventricular drain at a certain height and keeping it open to drain might have led to the low incidence of having ICP greater than $20 \mathrm{~mm} \mathrm{Hg}$ during the observation period. The authors also concluded that ICP elevation was not routinely associated with EVD closure for thrombolytic treatment. This finding has an important practical implication as we continue to learn about the efficacy of thrombolytic therapy for treating patients with severe IVH. The observation of intraventricular administration of UK or placebo not being associated with unwanted ICP elevation (both during the drug administration and 1-hour period of drain closure) may also provide additional benefits in properly designing other clinical trials that may involve injection of pharmacological agents into the ventricles. Another clinically pertinent point that was reiterated by this paper was that EVD was equally effective in controlling ICP in both the study arm and the placebo group, once again justifying the current indication and our understanding of EVD placement not only as a diagnostic but also therapeutic measure. The analysis of multiple ICP measurements in this cohort of severe IVH patients suggests that ICP may not be the only factor determining the harmful effect of IVH. Again, it is important to emphasize that these findings should not be used to argue for any delay in placing EVD in patients with severe IVH.

\section{KARGER}

Fax +41613061234 E-Mail karger@karger.ch www.karger.com
(อ) 2009 S. Karger AG, Base

$1015-9770 / 09 / 0274-0411 \$ 26.00 / 0$ 


\section{References}

$\checkmark 1$ Bhattathiri PS, Gregson B, Prasad KS, Mendelow AD; STICH Investi gators: Intraventricular hemorrhage and hydrocephalus after spontaneous intracerebral hemorrhage: results from the STICH trial. Acta Neurochir Suppl 2006;96:65-68.

-2 Steiner T, Diringer MN, Schneider D, Mayer SA, Begtrup K, Broderick J, Skolnick BE, Davis SM: Dynamics of intraventricular hemorrhage in patients with spontaneous intracerebral hemorrhage: risk factors, clinical impact, and effect of hemostatic therapy with recombinant activated factor VII. Neurosurgery 2006;59:767-774.

-3 Hemphill JC 3rd, Bonovich DC, Besmertis L, Manley GT, Johnston SC: The ICH score: a simple, reliable grading scale for intracerebral hemorrhage. Stroke 2001;32:891-897.

$\checkmark 4$ Hallevi H, Albright KC, Aronowski J, Barreto AD, Martin-Schild S, Khaja AM, Gonzales NR, Illoh K, Noser EA, Grotta JC: Intraventricular hemorrhage: anatomic relationships and clinical implications. Neurology 2008;70:848-852.
5 Hütter BO, Kreitschmann-Andermahr I, Gilsbach JM: Cognitive deficits in the acute stage after subarachnoid hemorrhage. Neurosurgery 1998;43:1054-1065.

6 Ziai WC, Torbey MT, Naff NJ, Williams MA, Bullock R, Marmarou A, Tuhrim S, Schmutzhard E, Pfausler B, Hanley DF: Frequency of sustained intracranial pressure elevation during treatment of severe intraventricular hemorrhage. Cerebrovasc Dis 2009;27:403-410.

\section{Kiwon Lee}

Neurology and Neurosurgery, Columbia University

College of Physicians and Surgeons

177 Fort Washington Ave, Mistein Hospital 8 Center, Room 300

New York, NY 10032 (USA)

Tel. +1 212305 7236, Fax +1 2123052792

E-Mailklee@neuro.columbia.edu

\section{Erratum}

The authors of the article 'Danqi Piantan Jiaonang Does Not Modify Hemostasis, Hematology, and Biochemistry in Normal Subjects and Stroke Patients' (Cerebrovasc Dis 2008;25:450-456) received a notification from the manufacturer regarding an error in the declaration of the ingredients of the study drug. The components of the study drug listed under the section 'Study Drug' on p. 451, third sentence, should read: Each capsule combines 9 herbal components [i.e. root of membranous milk vetch, red sage root, red peony root, rhizome of Ligusticum chuanxiong, root of thinleaf milkwort, rhizome of grassleaf sweetflag, root of Chinese angelica, safflower, peach seed (Prunus persica)] and 5 animal components (i.e. Whitmania pigra Whitman, Eupolyphaga seu Steleophaga, Buthus martensii Karsch, calculus bovis artifactus, cornu saigae tataricae). 